\title{
Aplicativo para Ganho de Vocabulário e Auxílio na Alfabetização destinado às Crianças com Transtorno do Espectro Autista
}

\author{
Maria Renata M. Gobbo ${ }^{1}$, Cinthyan Renata Sachs C. de Barbosa ${ }^{1}$, \\ Marcelo Morandini ${ }^{2}$, Fernanda Mafort $^{3}$ \\ ${ }^{1}$ Programa de Pós-Graduação em Ciência da Computação - Universidade Estadual de \\ Londrina (UEL) Londrina, PR - Brasil. \\ ${ }^{2}$ Escola de Artes, Ciências e Humanidades - Universidade de São Paulo (USP) \\ São Paulo, SP - Brasil. \\ ${ }^{3}$ Escola de Ciências da Vida - Pontifícia Universidade Católica do Paraná (PUCPR) \\ Londrina, PR - Brasil \\ \{mr.gobbol@gmail.com, cinthyan@uel.br, m.morandini@usp.br, \\ fernanda-mafort@hotmail.com \}
}

\begin{abstract}
Autism Spectrum Disorder (ASD) is a neurodevelopmental disorder that can impair, to some extent, communication, socialization and also present stereotyped and restricted behaviors. The objective of this article was to present test results from the ACA (Learning with Alternative Communication) application, which uses the images of the Daily Life Activities to teach naming of objects, alphabet, syllables, and words, and to verify if there was vocabulary gain and literacy aid growth, as well as its usability and gamification issues. The tests were carried out with 28 children diagnosed with mild and moderate ASD. The results showed that such App is effective in its performance, since children have gained vocabulary.
\end{abstract}

Resumo. Transtorno do Espectro Autismo (TEA) é um distúrbio de neurodesenvolvimento que pode comprometer, em algum grau, a comunicação, socialização e apresentar comportamentos estereotipados e restritos. $O$ objetivo deste artigo foi apresentar resultados dos testes do aplicativo ACA (Aprendendo com Comunicação Alternativa), que utiliza imagens de Atividades de Vida Diária para ensinar a nomeação de objetos, alfabeto, sílabas e palavras, e verificar se ocorreu ganho de vocabulário e auxílio à alfabetização, como também questões de usabilidade e gamificação. Os testes foram feitos com 28 crianças diagnosticadas com TEA de graus leve e moderado. Os resultados mostraram que tal aplicativo é eficaz no que promete, visto que as crianças ganharam vocabulário.

\section{Introdução}

De acordo com Ramdoos et al. (2011), o Transtorno do Espectro Autista (TEA) é um distúrbio do desenvolvimento neurológico de início precoce, caracterizado por comprometer a comunicação, habilidades sociais e comportamentos estereotipados ou 
VIII Congresso Brasileiro de Informática na Educação (CBIE 2019)

Anais do XXX Simpósio Brasileiro de Informática na Educação (SBIE 2019)

restritos. Essas crianças podem apresentar resistência às modalidades sensoriais, como lavagem e escovação do cabelo ou dos dentes, usar certos tipos de roupas, etc., gerando um grande prejuízo para a independência desses indivíduos (Mashal e Kasirer 2012).

PECS (Picture Exchange Communication System), que é um método de comunicação desenvolvido por meio de PCSs (Picture Communication Symbols) tem sido usado para crianças do espectro autista. Esse vem do campo da comunicação alternativa, a qual é englobada por uma área maior chamada de Tecnologia Assistiva, que tem como um de seus vários objetivos compensar, aliviar ou neutralizar uma incapacidade ou desvantagem gerada por alguma "deficiência" (Galvão Filho, 2009). Um dos seus campos trata-se de intervenções e serviços associados com a educação que realiza uma associação entre símbolo e atividade para ajudar crianças e adultos autistas ou com outros distúrbios de desenvolvimento a adquirir habilidades de comunicação.

Convém lembrar que pesquisas relacionadas ao ganho de vocabulário em crianças com TEA é de importância significativa, visto que se essas não forem estimuladas de forma correta podem acabar perdendo até mesmo o vocabulário já adquirido, salienta Bordini et al. (2014). Além disso, as crianças precisam saber nomear objetos para desenvolver leitura com compreensão e muitas têm dificuldade em relacionar um nome a um objeto (Whalon e Hart 2011).

A mesma importância deve ser dada às pesquisas relacionadas à motivação que a gamificação pode oferecer aos indivíduos com TEA. Coelho Neto, Blanco e Silva (2018) fizeram uma pesquisa em Anais do SBIE de 2007 a 2017 e não encontraram artigos que contemplassem o tema Gamificação e Autismo. A temática de usabilidade nos aspectos visuais e de comunicação também é de suma importância para o público autista, uma vez que há toda uma peculiaridade sensorial, de comportamento, etc.

Este artigo irá abordar questões de usabilidade, gamificação, assim como dados que apontam o aprendizado gerado com o uso de um aplicativo desenvolvido, o qual foi denominado ACA. Também foram apontadas características que podem interferir ou dificultar as coletas de dados para construção de um aplicativo para autistas. Isso poderá servir como ponto de partida para outras pesquisas envolvendo tecnologias para aumentar a comunicação e alfabetização desse público.

\section{Trabalhos relacionados}

Para o tratamento de indivíduos com TEA é necessária uma intervenção muito precoce, de caráter intensivo e multidisciplinar (Lima 2012). De acordo com Silva, Salgado e Raposo (2013), o uso de tecnologias para crianças com autismo pode auxiliar seu desenvolvimento, por isso alguns trabalhos serão apresentados aqui.

Bittencourt e Fumes (2016) apresentam o Sistema de Comunicação Alternativa para Letramento de Pessoas com Autismo (SCALA), no qual seu objetivo é auxiliar o letramento de pessoas com autismo. Possui o módulo de prancha de comunicação e outro para construção de histórias. As análises foram feitas com dois adultos, onde esses construíram histórias relacionadas com suas vidas.

O mTEA, apresentado por Silva, Soares e Benitez (2017), tem como objetivo criar um ambiente de ensino personalizado de habilidades básicas como formar ou sobrepor palavras e selecionar, nomear ou sequenciar figuras. O software foi 
VIII Congresso Brasileiro de Informática na Educação (CBIE 2019)

Anais do XXX Simpósio Brasileiro de Informática na Educação (SBIE 2019)

desenvolvido para ser utilizado em sala de aula, pois as atividades a serem feitas pelo indivíduo autista precisam ser pré-elaboradas por terceiros.

Cotidiano trata-se de um protótipo não testado com indivíduos autistas (Afranio Neto et al. 2017) que tem por objetivo utilizar conceitos de atividades diárias para aplicar no cotidiano dessas, levando em consideração não apenas os tipos de perturbações, mas também as intervenções educacionais existentes em uma tecnologia de apoio, que serviram de base para a concepção do aplicativo.

Outro aplicativo é o TEO (Moura et al. 2016), que embora não seja um jogo para alfabetização, trata-se de um modelo de jogos interativos. Ele foi construído para trabalhar o desenvolvimento cognitivo da criança com espectro autista e é dividido em várias categorias como: associação, raciocínio lógico-matemático, quebra-cabeça, exercícios de memorização, atividades de vida diária e localização.

Porém, a maioria desses trabalhos não leva em conta: i) realização de atividades simples que não precisam ser pré-elaboradas por terceiros; ii) utilização de figuras relacionadas com Atividades de Vida Diária(AVDs); iii) recomendações de design para indivíduos com TEA; iv) realização de testes com um grupo grande de indivíduos autistas; v) auxílio para ganho de vocabulário.

\section{Metodologia ACA - Aprendendo com Comunicação Alternativa}

A tecnologia utilizada para o desenvolvimento do aplicativo ACA (Aprendendo com Comunicação Alternativa) descrito neste trabalho foi o Construct 2, por ser compatível com vários dispositivos móveis que se encontram no mercado. $\mathrm{O}$ app já está disponível na plataforma do Google Play e pode ser usado para ganho de vocabulário e auxílio à alfabetização de forma gratuita. Todas as figuras presentes no mesmo estão relacionadas com AVDs dessas crianças e foram tiradas do portal ARASSAC ${ }^{1}$.

O ACA foi baseado no TEACCH (Treatment and Education of Autistic and Related Communication Handcapped Children), que trata o autismo como um processo neurobiológico e visa melhorar a adaptação do indivíduo via exploração das habilidades e interesses por meio do ensino individualizado estruturado e fortemente baseado nas teorias comportamental e cognitivas empíricas. Trabalha essencialmente com a estruturação do tempo, atividades, materiais e ambientes usados pela criança visando compensar déficits típicos do TEA e proporcionar ganhos significativos para o convívio social.

O ABC Autismo (Farias, Silva e Cunha 2014) utiliza o método TEACCH que traz palavras descontextualizadas de um tema específico e não se atenta à sensibilidade auditiva das crianças. Assim, no ACA optou-se abordar as AVDs e palavras relacionadas a elas. ACA se difere também do SCALA (Bittencourt e Fumes 2016) em nível de instrução esperada pelo usuário. Nesse, para a utilização do módulo de construção de histórias, deve-se estruturar uma narrativa, o que é muito complexo para indivíduos com TEA, enquanto naquele optou-se em não trabalhar com frases e sim com questões relacionadas à morfologia.

\footnotetext{
1 ARASAAC: Portal Aragonês de Comunicação Aumentativa e Alternativa, http://www.arasaac.org/ Acesso em: 12 jun. 2019
} 
VIII Congresso Brasileiro de Informática na Educação (CBIE 2019)

Anais do XXX Simpósio Brasileiro de Informática na Educação (SBIE 2019)

$\mathrm{O}$ primeiro nível do ACA tem por objetivo ensinar crianças com TEA a distinguir formas e é baseado no nível II do TEACCH descrito em Fonseca e Ciola (2014). O segundo nível (Figura 1a) foi baseado no nível III do referido método e tem como objetivo auxiliar a leitura com compreensão e aumentar o vocabulário dessas crianças. O terceiro nível ensina as letras do alfabeto (Figura 1b), enquanto que o quarto nível, as sílabas simples (Figura 1c) sendo baseado no nível IV do TEACCH. O quinto nível auxilia na associação de imagem e palavra escrita (Figura 1d). O aplicativo também conta com elementos de gamificação como pontos, medalhas e troféus.

A metodologia de alfabetização aqui utilizada incentiva o ensino das habilidades rudimentares pelo modelo de equivalência de estímulos (Gomes 2015). É possível simular aprendizagem ensinando aos indivíduos a relacionar palavras verbais (conjunto A) com a figura (conjunto B) correspondente. Com isso, aprende-se a relacionar a palavra falada com o objeto concreto e são estabelecidas as relações entre os elementos do conjunto A com o B (relações $\mathrm{AB}$ ). Logo após pode-se acrescentar mais um tipo de estímulo, com as palavras escritas (conjunto $C$ ). Assim, serão ensinadas as relações de palavras ditas (conjunto A) com a palavra escrita (conjunto C), tendo as relações AC.
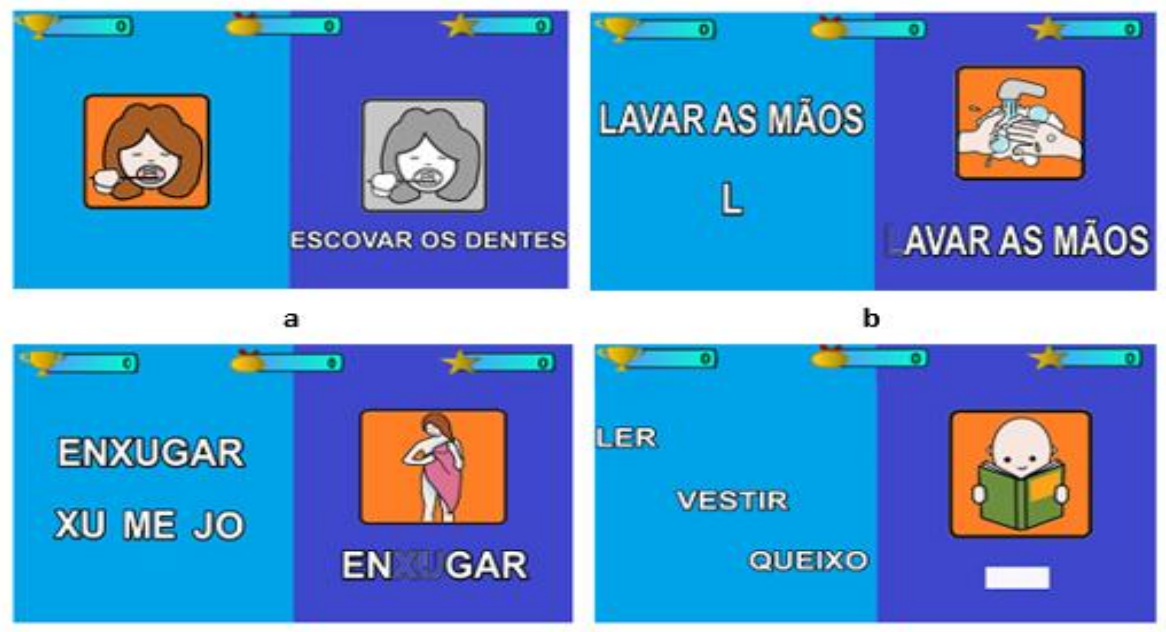

c

d

Figura 1: Exemplo do Aplicativo ACA

Os métodos de design interativo usados foram os de Preece, Rogers e Sharp (2005) que auxiliam a criar experiências de usuário visando facilitar a interação. Foram empregadas as recomendações de Britto (2016) para o design de tela multi-toque. Essas indicam boas práticas para a construção de ferramentas para autistas em relação à utilização de cores, linguagem, imagens, interfaces, entre outras.

A sequência de letras ensinada segue o método fônico descrito por Capovilla e Capovilla (2004). ACA pode ser utilizado para todos os graus do TEA. Os de alto funcionamento poderão ser alfabetizados rapidamente por possuírem facilidade em memorização e os de baixo nível poderão ser auxiliados nesse processo de maneira mais lenta por meio de requisitos prévios que foram levados em conta na sua construção. 
VIII Congresso Brasileiro de Informática na Educação (CBIE 2019)

Anais do XXX Simpósio Brasileiro de Informática na Educação (SBIE 2019)

\section{Resultados e discussões}

A presente pesquisa foi desenvolvida por meio de uma abordagem quantitativa, bem como qualitativa (Ludke e André 2011), pois os dados a serem coletados serão predominantemente descritivos desempenhando um papel fundamental tanto no processo de obtenção dos dados quanto na análise dos resultados. Em Santos (2016) salienta-se também a escassez de estudos sobre a maneira como se articulam pensamento e linguagem na constituição desses sujeitos (autistas). Tal é a grande motivação deste trabalho e da metodologia empregada.

Os testes foram aplicados de forma igual a todos os participantes desta pesquisa. Antes de começarem a utilizar o ACA, as crianças passaram por um pré-teste no notebook que respondiam ou apontavam questionamentos sobre as imagens/palavras/sílabas/letras presentes no app para verificar se elas já as conheciam previamente, sendo anotadas suas respostas em um questionário impresso. Também foram extraídas informações sobre os perfis dos usuários com os terapeutas das crianças ou professores da educação especial, referentes ao grau de autismo, se possuíam linguagem verbal e/ou oral, se relacionavam com seus pares, etc. Durante o uso do aplicativo foram coletadas informações sobre as dificuldades e usabilidade e após foi feito um pós-teste no notebook para verificar se houve ganho de vocabulário e avanços na alfabetização.

Os testes foram realizados com crianças diagnosticadas com TEA tanto de uma escola pública como de uma clínica particular de psicologia (ambas especializadas em autismo) e aconteceram nas salas onde as crianças já tinham costume de realizar atividades educativas e contaram com um smartphone contendo o aplicativo ACA previamente instalado e foi utilizado um roteiro impresso para coleta de dados. $\mathrm{O}$ tamanho da amostra era de 44 indivíduos com autismo, com idade de 2 a 15 anos, porém apenas 28 desses possuíam requisitos mínimos para participar da pesquisa, os outros 16 não conseguiram ficar sentados, por exemplo, durantes os testes ou não respondiam às demandas. Isso foi feito de forma legal, com autorização do Comitê de Ética em Pesquisa em Serem Humanos da Universidade Estadual de Londrina e por meio do Termo de Consentimento Livre e Esclarecido dos responsáveis.

Aprendizado gerado durante a utilização do aplicativo: a Tabela 1 conta com os dados quantitativos coletados durante o pré-teste e após usar o aplicativo (pós-teste), exibidos em Gobbo (2019). Indivíduos A's e B's são, respectivamente, da escola pública e da clínica particular. É possível ver a idade de cada participante na tabela, seu nível de autismo, número de acertos em seu pré e pós-teste, nos quais foram testadas 55 imagens, 55 palavras, 63 sílabas e 24 letras do alfabeto. Alguns participantes não conseguiram utilizar todos os níveis do aplicativo ACA por não terem os pré-requisitos necessários (conhecimento prévio das letras, sílabas e palavras) ou não terem vontade de continuar utilizando o app. Pode-se observar na referida tabela que as medidas de pósteste foram superiores as de pré-teste e que os participantes tiveram bons resultados no ganho de vocabulário. Verifica-se que as crianças/adolescentes aprenderam novas imagens, pois aumentaram a porcentagem de acertos no pós-teste. Já com relação à alfabetização no ACA, não se espera que crianças de 3 a 5 anos possuam conhecimento de sílabas e palavras, pois geralmente tal aprendizado acontece após os 5 anos.

Gamificação na motivação de crianças com TEA: Embora o aplicativo ACA 
VIII Congresso Brasileiro de Informática na Educação (CBIE 2019)

Anais do XXX Simpósio Brasileiro de Informática na Educação (SBIE 2019)

siga as peculiaridades de aprendizagem dos indivíduos com autismo, esse deve procurar outras formas de atrair tal público para sua utilização sem haver desmotivação, como aconteceu algumas vezes durante os testes. $\mathrm{O}$ uso de pontos, medalhas e troféus não foi previamente apresentado aos usuários e esperava-se que eles notassem esses elementos, porém, apenas 4 das 28 crianças perceberam a pontuação. Isso pode ter acontecido por falta do aplicativo destacá-los ou por esses elementos não serem ainda suficientes para motivar autistas. Uma alternativa seria utilizar elementos imersivos, fazendo com que os autistas se sintam dentro do jogo.

Tabela 1 - Tabela com os dados do pré e pós-teste

\begin{tabular}{|c|c|c|c|c|c|c|c|c|c|c|}
\hline & Idade & Nível & $\begin{array}{l}\text { Imagens } \\
\text { (pré) }\end{array}$ & $\begin{array}{l}\text { Imagens } \\
\text { (pós) }\end{array}$ & $\begin{array}{l}\text { Letras } \\
\text { (pré) }\end{array}$ & $\begin{array}{l}\text { Letras } \\
\text { (pós) }\end{array}$ & $\begin{array}{l}\text { Sílabas } \\
\text { (pré) }\end{array}$ & $\begin{array}{l}\text { Sílabas } \\
\text { (pós) }\end{array}$ & $\begin{array}{l}\text { Palavras } \\
\text { (pré) }\end{array}$ & $\begin{array}{l}\text { Palavras } \\
\text { (pós) }\end{array}$ \\
\hline $\mathbf{A} 1$ & 15 & Moderado & 19 & 33 & - & - & - & - & - & - \\
\hline A2 & 15 & Leve & 52 & 54 & 22 & 22 & 66 & 66 & 55 & 55 \\
\hline A3 & 14 & Leve & 52 & 55 & 22 & 22 & 63 & 63 & - & - \\
\hline A4 & 15 & Moderado & 44 & 52 & 11 & 11 & - & - & - & - \\
\hline A5 & 9 & Leve & 53 & 55 & 21 & 22 & - & - & - & - \\
\hline A6 & 13 & Moderado & 49 & 55 & 22 & 22 & 63 & 63 & - & - \\
\hline A7 & 12 & Moderado & 21 & 36 & 19 & 22 & - & - & - & - \\
\hline A8 & 8 & Leve & 39 & 46 & 22 & 22 & - & - & - & - \\
\hline B1 & 8 & Leve & 29 & 48 & 22 & 22 & 63 & 63 & 55 & 55 \\
\hline B2 & 10 & Leve & 48 & 52 & 22 & 22 & 61 & 62 & 55 & 55 \\
\hline B3 & 8 & Leve & 47 & 53 & 22 & 22 & 62 & 63 & 55 & 55 \\
\hline B4 & 14 & Leve & 49 & 55 & 22 & 22 & 63 & 63 & 55 & 55 \\
\hline B5 & 6 & Leve & 37 & 51 & 22 & 22 & 63 & 63 & 55 & 55 \\
\hline B6 & 3 & Leve & 10 & 23 & 19 & 22 & - & - & - & - \\
\hline B7 & 8 & Moderado & 25 & 34 & 22 & 22 & 47 & 60 & 19 & 29 \\
\hline B8 & 8 & Leve & 36 & 41 & 21 & 22 & 57 & 57 & 20 & 41 \\
\hline B9 & 3 & Leve & 34 & 46 & 15 & 16 & - & - & - & - \\
\hline B10 & 10 & Leve & 44 & 55 & 22 & 22 & 63 & 63 & 55 & 55 \\
\hline B11 & 5 & Leve & 44 & 47 & 21 & 22 & - & - & - & - \\
\hline B12 & 3 & Leve & 42 & 50 & 20 & 22 & - & - & - & - \\
\hline B13 & 3 & Leve & 32 & 44 & 22 & 22 & - & - & - & - \\
\hline B14 & 7 & Leve & 52 & 55 & 22 & 22 & 63 & 63 & 55 & 55 \\
\hline B15 & 3 & Leve & 36 & 38 & 22 & 22 & - & - & - & - \\
\hline B16 & 3 & Leve & 18 & 25 & 18 & 20 & - & - & - & - \\
\hline B17 & 4 & Leve & 35 & 50 & 22 & 22 & - & - & - & - \\
\hline B18 & 11 & Leve & 40 & 48 & 22 & 22 & - & - & - & - \\
\hline B19 & 3 & Leve & 31 & 44 & 20 & 22 & - & - & - & - \\
\hline B20 & 4 & Leve & 26 & 55 & 20 & 21 & - & - & - & - \\
\hline
\end{tabular}

Dados de usabilidade: durante os testes com o app pôde-se observar que as crianças de 3 anos ou mais conseguiram entender as atividades da ferramenta. Algumas descobriram outras atividades, tal como clicar em uma imagem para reproduzir um som. 
VIII Congresso Brasileiro de Informática na Educação (CBIE 2019)

Anais do XXX Simpósio Brasileiro de Informática na Educação (SBIE 2019)

Outro ponto observado durante os testes foi a falta de interesse de algumas crianças após certo tempo de uso do aplicativo, principalmente as de 3 a 5 anos, independentemente do nível do autismo, pois essas tendem a se cansar mais facilmente.

De acordo com Kern et al. (2006), ao construir aplicativos para autistas deve-se ter a preocupação com as cores utilizadas no mesmo que devem ser bastante atrativas. Para garantir uma interface que atente à sensibilidade visual de autistas, a cor de fundo utilizada foi adequada para que haja uma distinção entre os itens e para que se possa diferenciar conteúdos e relacionar informações similares. Assim, quanto aos aspectos visuais do ACA, verificou-se que $85,2 \%$ das crianças optaram pelo background com o fundo escuro. Essa informação foi obtida de maneira simples, mostrando a interface com tom de azul escuro e outra com tom claro e foi pedido que as crianças escolhessem quais dos dois preferiam. Acredita-se que as cores na tela do celular não causem desconfortos visuais na maioria das crianças com autismo, porém só foram utilizadas cores frias nos testes. Não foi verificado o uso de cores quentes, como vermelho ou laranja, uma vez que alguns autistas são bem sensíveis a essas.

Outro ponto nos aspectos visuais é a escolha por utilização de imagens que não contenham muitas informações. Na Figura 2 é mostrada a imagem de um quadro à esquerda que possui um urso, uma mesa e uma casa. Uma das crianças, ao ver a imagem no pré-teste, disse que aquilo era um urso e não um quadro. Então, optou-se por retirar as informações impertinentes, conforme o quadro no lado direito. Deve-se lembrar que autistas geralmente se fixam nos detalhes, então enquanto mais clean a figura melhor.
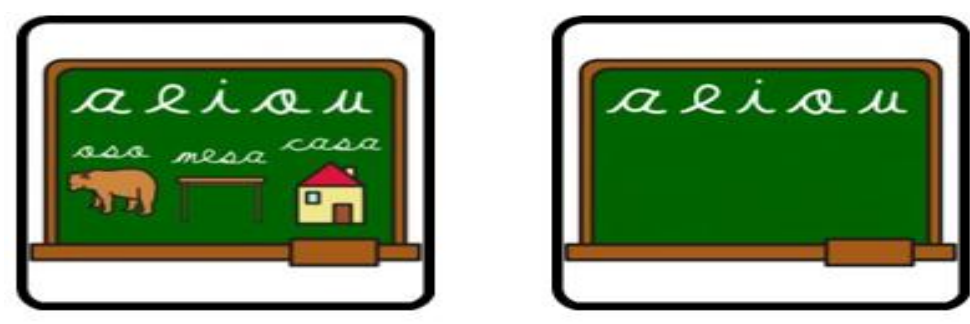

Figura 2 - Imagem com muitas informações à esquerda e essa alterada à direita

Para a criação do ACA foi analisado também o software ABC Autismo (Farias, Silva e Cunha 2014) e percebeu-se que esse não se atentava à sensibilidade auditiva de crianças com autismo, possuindo vários sons altos e desnecessários que eram acionados quando passava de fase ou fazia uma interação errada. Isso não acontece no ACA, pois estímulos sonoros só vêm com as letras, sílabas e palavras, bastando clicar em cima dessas e o áudio é acionado pronunciando-as. Nas atividades da fase cinco do aplicativo, uma palavra é falada para que o usuário possa procurar sua escrita correspondente apresentando também sua imagem. Com as fases do ACA é possível auxiliar as crianças na aquisição de vocabulário, pois esse se relaciona à capacidade de entender o conceito e significado de palavras, como descrito por Nalom, Soares e Cárnio (2015). O nível de dificuldade aumenta e é acionado o emparelhamento multimodelo, com figuras ou letras/sílabas/palavras diferentes para associar com seu par correspondente.

As legendas e o reforço sonoro no ACA puderam auxiliar no processo de comunicação e ganho de vocabulário dessas crianças, principalmente quando elas repetiam as palavras emitidas pelo aplicativo. O participante A4 mesmo sem ser pedido, repetia todas as palavras que ouvia. Já B6, embora não conseguisse falar perfeitamente a 
VIII Congresso Brasileiro de Informática na Educação (CBIE 2019)

Anais do XXX Simpósio Brasileiro de Informática na Educação (SBIE 2019)

palavra, tentava emitir o mesmo som. O participante A7 quando não conseguia ouvir a reprodução do som por motivos de barulho externos colocava o smartphone no ouvido. Os estímulos sonoros associados aos estímulos visuais ajudaram as crianças a compreenderem o que a imagem significava. Isso ficou claro no momento em que algumas crianças utilizavam o aplicativo e entoavam em tom de entendimento um " $A h$, estômago!" ao invés de só repetir a palavra reproduzida pelo aplicativo.

Em relação ao conhecimento prévio das imagens presentes no aplicativo foi possível verificar que algumas crianças não identificaram imagens de ações, como escovar os dentes ou mesmo brincar. Isso ocorreu em diversos testes, principalmente em crianças com maiores comprometimentos. Com isso ocorreu uma dificuldade de algumas crianças reconhecerem AVDs durante a aplicação do pré-teste. Conclui-se que, possivelmente, essas tenham dificuldade de reconhecer imagens contendo ações.

O participante A6 comprova que crianças com autismo podem não responder bem aos meios tradicionais de educação, mas podem realizar com sucesso atividades nos meios digitais. Segundo a professora, esse não realiza nenhuma atividade de escrita ou leitura na sala de aula e apresenta comportamento agressivo quando lhe é imposto, porém usando o smartphone realizou todas as atividades propostas sem hesitar, reclamar ou ter qualquer comportamento agressivo.

Os participantes B7 e B8 eram os participantes com mais de 8 anos que mais apresentavam comprometimentos de comportamento e interação, porém foram os que apresentaram mais ganhos em geral. Durante os testes foram presenciadas algumas características do TEA em vários participantes que dificultaram as coletas de dados:

Hiperatividade ou passividade: crianças que não param sentadas foi um problema na coleta, principalmente as mais novas. Muitas preferiam ficar correndo ou pulando e essas foram excluídas da pesquisa. Em relação à passividade, tivemos apenas o caso de uma criança que não respondeu às instruções verbais e que também foi excluída.

Resistência em mudar a rotina: a aplicação dos testes nessas crianças implicava em uma mudança brusca de rotina e isso em algumas gerava comportamentos inadequados, como autoagressão ou agressão a outras pessoas. Um exemplo foi um participante de 13 anos que se irritou no começo do pré-teste. Toda hora esse fechava a tela do notebook e gritava. O teste foi interrompido por um profissional da psicologia, o qual acreditava que esse poderia agredir outra pessoa a qualquer momento.

Agir como se estivesse surdo: não responder às inscrições verbais foi um problema durante a coleta de dados. Muitas crianças autistas parecem não ouvir quando é pedido para realizarem algo que não é de seu interesse.

Outra constatação no decorrer do trabalho foi fazer com que os pais entendessem que a utilização do aplicativo traria vantagens não apenas durante o(s) dia(s) de testes, mas sim deve ser utilizado diariamente pelos participantes em outros ambientes (casa, terapias, etc.). Como em qualquer outra forma de ensino, a aprendizagem acontece lentamente e de forma gradual.

Outra característica que não dificultou a coleta de dados, mas que foi observada:

Ausência de resposta aos métodos normais de ensino: um dos participantes de 13 anos que não realiza atividades em sala de aula e em sua ficha estava escrito que ele era 
VIII Congresso Brasileiro de Informática na Educação (CBIE 2019)

Anais do XXX Simpósio Brasileiro de Informática na Educação (SBIE 2019)

não verbal, baixa interação social e não totalmente alfabetizado, porém completou todos os níveis do aplicativo demonstrando interesse.

\section{Conclusões}

A proposta deste artigo foi validar o aplicativo ACA com crianças/adolescente de 3 a 15 para verificar algumas questões, como ganho de vocabulário, auxílio à alfabetização, gamificação e aspectos de usabilidade. O aplicativo foi eficaz em sua proposta, visto que todos os participantes da pesquisa tiveram um ganho de vocabulário. Em relação ao auxílio da alfabetização, os que conseguiram concluir $100 \%$ dos níveis, já estavam alfabetizados, porém acredita-se que a prática pode potencializar o entendimento dessas crianças em relacionar imagens com suas AVDs.

Outro ponto de destaque foi o conjunto de atividades não realizáveis em smartphones, como o pré e pós-teste, que foram mais difíceis de serem feitos quando comparadas com o uso do aplicativo em si. Preferiram smartphone a notebook.

A pesquisa também tentou verificar a eficácia da gamificação como instrumento de motivação, mas a falta de imersão nos elementos gamificados pode ter influenciado os resultados. Uma hipótese é que isso pode ser resolvido com a inserção de ranking mundial, realidade aumentada ou até mesmo desafios. Já dados de usabilidade colhidos poderão servir de alicerce para futuras aplicações de smartphones.

Conclui-se que o ACA pode auxiliar a alfabetização e ganho de vocabulário de indivíduos com autismo, pois possui recursos para isso. Porém, esse não substitui os meios básicos de alfabetização, mas sim auxilia no ganho de vocabulário e deve ser usado sempre que possível para melhorar a eficácia. Todo esse processo é uma continua construção de saberes, por isso cabe a cada profissional, independente da área, ajudar da maneira que lhe cabe, quebrando barreiras, superando obstáculos e buscando colaborar para a melhoria da vida dessas pessoas.

\section{Referências}

Afranio Neto, Rufino, H., Nakamoto, P., Palis, R. e Beira, D. (2017) "Cotidiano: um software para auxiliar crianças autistas em suas atividades diárias". XXVIII Simpósio Brasileiro de Informática na Educação. p.404-413.

Bittencourt, I. e Fumes, N. (2016) "A tecnologia assistiva SCALA na promoção de narrativas de sujeitos com Transtorno do Espectro Autista sobre as suas experiências escolares e o autismo". XXVII Simpósio Brasileiro de Informática na Educação. p.767-776.

Bordini, R. A., Freitas, P. A. G., Fonseca, L. F., Nunes, A. P. A, Santiago, D. L., Otsuka, J. L., Beder, D. M., Santiago, G. L. A. e Oliveira, M. R. G. (2014) "Processo de Design de um jogo eletrônico para o aprendizado de Teclado Musical". XIII Computer Games and Digital Entertainment, p.139-147.

Britto, T. C. P. (2016) GAIA: uma proposta de guia de recomendações de acessibilidade web com foco em aspectos do autismo. Dissertação de Mestrado, Universidade Federal de São Carlos, São Carlos, SP.

Capovilla, A. G. S. e Capovilla, F. C. (2004) Alfabetização: método fônico, Memnon.

Coelho Neto, J., Blanco, M. B. e Silva, J. A. (2018) "Gamificação e Discalculia: uma revisão nos Anais do Simpósio Brasileiro de Informática da Educação". 
VIII Congresso Brasileiro de Informática na Educação (CBIE 2019)

Anais do XXX Simpósio Brasileiro de Informática na Educação (SBIE 2019)

I Encontro Paranaense de Tecnologias em Educação Matemática, p.1-15.

Farias, E. B., Silva, L. W. C. e Cunha, M. X. C. (2014). “ABC Autismo: um aplicativo móvel para auxiliar na alfabetização de crianças com autismo baseado no Programa TEACCH”. X Simpósio Brasileiro de Sistemas de Informação, p.458-469.

Fonseca, M. E. G. e Ciola, J. C. B. (2014) Vejo e Aprendo: Fundamentos do Programa TEACCH: o ensino estruturado para pessoas com autismo, Book Toy.

Galvão Filho, T. A. (2009) “A Tecnologia Assistiva: de que se trata?”. In Conexões: educação, comunicação, inclusão e interculturalidade, pages 207-235. Redes.

Gobbo, M. R. M. (2019) Aplicativo ACA (Aprendendo com Comunicação Alternativa) para Crianças com Transtorno do Espectro Autista. Dissertação de Mestrado, Universidade Estadual de Londrina, Londrina, PR.

Gomes, C. G. S. (2015) Ensino de leitura para pessoas com autismo, Appris.

Kern, J. K., Trivedi, M. H., Garver, C. R., Grannemann, B. D., Andrews, A. A., Savla, J. S., Johnson, D. G., Mehta, J. A. and Schroeder, J. L. (2006) The pattern of sensory processing abnormalities in autism. In Autism, vol.10, n.5, pages 480-494.

Lima, C. B. (2012) Perturbações do Espectro do Autismo: Manual Prático de Intervenção, Lidel.

Ludke, M. e André, M. E. D. A. (2011). Pesquisa em educação: abordagens qualitativas. In Em Aberto, vol. 5, n. 31.

Mashal, N. and Kasirer, A. (2012) Principal component analysis study of visual and verbal metaphoric comprehension in children with autism and learning disabilities In Research in developmental disabilities, vol. 33, pages 274-282.

Moura, D., Oliveira Filho, D. L. S., Laertius, D., Silva, A. J. G., Paiva, P., Sales, T., Cavalcante, R. e Queiroz, F. (2016) “TEO: Uma suíte de jogos interativos para apoio ao tratamento de crianças com autismo". XXVII Simpósio Brasileiro de Informática na Educação, p.627-636.

Nalom, A. F. O., Soares, A. J. C. e Cárnio, M. S. (2015). A relevância do vocabulário receptivo na compreensão leitora. In: CoDAS, vol. 27, n. 4, pages 333-338.

Preece, J., Rogers, Y. e Sharp, H. (2005) Design de interação, Bookman.

Ramdoss, S., Mulloy, A., Lang, R., O’Reilly, M., Sigafoos, J., Lancioni, G., Didden, R. and El Zein, F. (2011) "Use of computer-based interventions to improve literacy skills in students with autism spectrum disorders: A systematic review". In: Research in Autism Spectrum Disorders, vol. 5, n. 4, pages 1306-1318.

Santos, E. C. (2016) Linguagem Escrita e a Criança com Autismo, Appris, $1^{\text {a }}$ Edição.

Silva, M. G. F., Salgado, L. C. e Raposo, A. B. (2013) "Metáforas de Perspectivas Culturais na (re) definição de padrões de colaboração de um jogo de multi-toque para usuários com autismo". In: 12th Brazilian Symposium on Human Factors in Computing Systems, p.112-121.

Silva, M., Soares, A. e Benitez, P. (2017) “Ambiente Digital para Ensino e Acompanhamento Personalizado de Estudantes com Autismo: proposta com Uso de Dispositivos Móveis". In: XXVIII Simpósio Brasileiro de Informática na Educação. p.1047-1056.

Whalon, K. J. and Hart, J. E. (2011) Children with autism spectrum disorder and literacy instruction: An exploratory study of elementary inclusive settings. In: Remedial and Special Education, vol. 32, n. 3, pages 243-255. 